\title{
Cognitive Impairment Predicts Fatal Incident Stroke: Findings from a National Sample of Older Adults
}

\author{
Philippa J. Clarke, PhD, ${ }^{*}$ Vanessa Blount, $M S c,{ }^{\dagger}$ and Angela Colantonio, $P h D^{\ddagger \mathcal{S}}$
}

OBJECTIVES: To investigate the effect of cognitive impairment on fatal and nonfatal incident stroke in older adults.

DESIGN: A large, national, prospective, population-based study of a representative cohort of older Canadians followed over a 10 -year period.

SETTING: Secondary analyses were conducted using data from the Canadian Study of Health and Aging, a population-based study of older adults followed prospectively from 1991 to 2001.

PARTICIPANTS: Nine thousand four hundred fifty-one adults aged 65 and older who had not previously been diagnosed with stroke at baseline (in 1991).

MEASUREMENTS: In addition to known risk factors, the independent contribution of cognitive function (diagnosed in a clinical examination) was examined as a risk for stroke in older adults.

RESULTS: Multinomial logistic regression analyses showed that cognitive impairment was associated with twice the odds of fatal incident stroke, controlling for wellestablished risk factors.

CONCLUSION: This study provides further evidence for the need to consider cognitive function in relation to stroke risk in older populations. J Am Geriatr Soc 59:1490-1496, 2011.

Key words: stroke; risk factors; cognitive function; Canadian Study of Health and Aging

Tn the United States, Canada, and much of the developed world, stroke is one of the three leading causes of death

From the *Institute for Social Research, University of Michigan, Ann Arbor, Michigan; ${ }^{\dagger}$ Ontario Ministry of Health and Long-Term Care, Toronto, Canada; and ${ }^{\ddagger}$ Toronto Rehabilitation Institute and Department of Occupational Science and Occupational Therapy, Graduate Department of Rehabilitation Science and ${ }^{\mathbb{S}}$ Dalla Lana School of Public Health, University of Toronto, Toronto, Canada.

Address correspondence to Philippa Clarke, Institute for Social Research, University of Michigan, 426 Thompson Street, Ann Arbor, MI 48104. E-mail: pjclarke@umich.edu

DOI: $10.1111 / j .1532-5415.2011 .03494 . x$ and a major cause of long-term disability. ${ }^{1}$ The prevalence of stroke increases rapidly with age. ${ }^{2,3}$ For older adults who survive the initial onset, a stroke can have a substantial effect on independence, quality of life, and social relationships. ${ }^{4,5}$ Reducing the incidence of stroke in later life requires the scientific identification of risk factors in older adult populations.

Important vascular risk factors include high blood pressure, cigarette smoking, and diabetes mellitus. ${ }^{6-8}$ Other identified risk factors include physical inactivity, excessive alcohol use, obesity, heart disease, and Parkinson's disease. $^{9-12}$ African Americans and Hispanics have higher rates of first stroke than whites, and men have higher incidence rates than women. ${ }^{13}$ Stroke incidence and mortality are higher in people with less education, whereas social supports may reduce the risk of stroke. ${ }^{14,15}$ Measures of physical function or frailty have also been found to be predictors of stroke in older adults, ${ }^{16}$ perhaps because impairments in physical function develop in the preclinical phases before stroke onset, yet most of this evidence comes from geographically constrained samples (e.g., Framingham Heart Study, Atherosclerosis Risk in Communities Study (ARIC), Baltimore Longitudinal Study of Aging), not from nationally representative studies focusing exclusively on older adult populations. As a result, generalizable stroke risk factors specific to the older population may not be fully understood.

There is evidence to suggest that risk factors for stroke are not always equivalent across age groups. ${ }^{17-19}$ Cognitive impairment, in particular, has been identified as a potentially unique risk factor in older adults. ${ }^{20-24}$ Data from the Established Population for Epidemiologic Studies of the Elderly (EPESE) indicate that older adults with poor cognitive function had twice the risk of stroke over a 3-year period. $^{21}$ This relationship was replicated with data from the Hispanic EPESE. ${ }^{20}$ A study using data from ARIC did not find a relationship between cognitive impairment and stroke incidence, most likely because the sample was restricted to a somewhat younger population (aged 48-67), ${ }^{22}$ but more-recent research using the same data found cognitive function to be an independent risk factor when stroke was pooled with all other cardiovascular outcomes. ${ }^{24}$

Given these inconsistent findings, the purpose of this research was to investigate the effect of clinically diagnosed 
cognitive impairment as an independent risk factor for fatal and nonfatal incident stroke in a large, national, prospective, population-based study of a representative cohort of older Canadians followed over a 10 -year period.

\section{METHODS}

\section{Data}

Secondary analyses were conducted with data from the Canadian Study of Health and Aging (CSHA), a population-based epidemiological study of dementia in 10,263 older adults followed prospectively over a 10 -year period (1991-2001). The first wave of the study (CSHA-1) began in 1991 with a representative sample of community $(\mathrm{n}=9,008)$ and institutional-dwelling $(\mathrm{n}=1,255)$ Canadians aged 65 and older from 36 urban and surrounding rural areas in all 10 Canadian provinces. A second wave of the study (CSHA-2) was conducted 4 years later (1995/96) when 9,265 surviving participants from the first wave were recontacted for follow-up. A third wave in 2001/02 (CSHA3 ) followed 5,456 surviving participants who were not diagnosed with dementia at CSHA-2.

Trained interviewers surveyed community-dwelling participants about their health, disability, and social circumstances using a standardized questionnaire (administered face-to-face in English or French). Participants were also given a screening test for cognitive impairment (the Modified Mini-Mental State Examination $(3 \mathrm{MS})^{25}$ ) and were referred (when their $3 \mathrm{MS}$ score was $<78$ ) for a further clinical assessment during which trained nurses, psychometrists, neuropsychologists, and physicians performed extensive tests to ascertain the diagnosis of dementia and cognitive impairment. Participants from the institutional sample (and a random subset from the community sample who did not screen positive on the 3MS) went directly to the clinical assessment. Further details on the study methods can be found elsewhere. ${ }^{26}$ The ethics review boards in each of the 18 study centers approved the procedures followed in CSHA, and all participating participants gave informed consent.

\section{Measures}

\section{Outcome Variables}

This study focused on two outcomes: nonfatal and fatal incident stroke. For community-dwelling participants, information on stroke incidence was collected through a self-report question: "Have you experienced a stroke or the effects of a stroke in the past year?" For participants referred to the clinical examination, a nurse or physician collected information on stroke occurrence during the clinical history. When participants had self-reported and clinical data (for community-dwelling participants referred to a clinical examination), the clinical report on stroke was used. Information on stroke mortality across the 10 years of the study was obtained from death certificates at the Office of the Registrar General in each province (underlying cause of death listed as International Classification of Diseases, Ninth Revision code 431 (cerebral hemorrhage), 433-434 (cerebral infarction), 436.0-437.1 or 437.9 (ill-defined cerebrovascular disease), or 438.0-438.9 (late effects of cerebrovascular disease). A stroke was considered to be an incident stroke if there was no mention of stroke in the previous waves, and the analyses are restricted to respondents without preexisting stroke at CSHA-1.

\section{Predictor Variables}

To the extent that they were available in the data, health and sociodemographic risk factors were examined as predictors of stroke. Information on heart disease, diabetes mellitus, hypertension, and Parkinson's disease was collected at each wave (through self-report in the community sample or clinical history and examination in the clinical sample). Cognitive impairment was based on a diagnosis of dementia or cognitive impairment without dementia (CIND) at the clinical examination. Physical disability was assessed through a count of the number of activities in which respondents report difficulty; seven items ask about basic self-care activities of daily living (ADLs) (e.g., bathing, dressing), and seven items probe difficulty with morecomplex activities (e.g., shopping, meal preparation). An overall disability score is created by summing the number of activities for which the respondent reported difficulty.

Information on sex, race (white vs black or other race), age $(67-75,76-85, \geq 86)$, and educational level $(<$ high school education, high school diploma, or $\geq$ college degree) was also included. In addition, information on marital status, as a measure of social support, was collected at each wave (married or partnered vs separated, divorced, widowed, or never married). Information on other important risk factors, including smoking, alcohol use, diet, and obesity, were not collected for all participants.

\section{Statistical Analyses}

Multinomial logistic regression was used to determine the relationship between stroke outcomes (no stroke, incident nonfatal stroke, incident fatal stroke), time-invariant sociodemographic factors, and time-varying factors (marital status, health conditions, cognitive function, and physical disability). Models regress stroke outcomes at a given wave on risk factors at the previous wave, controlling for year of assessment. Generalized estimating equations were used to account for the correlation between observations for each participant in the repeated-measures design. Models also include a dummy variable to account for the method of stroke outcome assessment (clinic- vs self-reported stroke) as a potential confounder. Statistical significance was assessed using a two-tailed alpha of .05. All statistical analyses were performed using SAS version 9.2 for Windows (SAS Institute, Inc., Cary, NC) using weighted data to correct for the oversampling of the original CSHA-1 sample by age and region.

\section{RESULTS}

Table 1 summarizes the sociodemographic and health characteristics of the study sample. On average, respondents were aged $73.6 \pm 6.8 ; 58 \%$ were female. At baseline, 812 participants $(7.9 \%$ of the study sample) had already experienced a stroke and were dropped from subsequent analyses, leaving 9,451 eligible participants. During the 10-year follow-up period, 873 incident strokes occurred (701 nonfatal, 172 fatal), with an overall incidence of 9.3 per 1,000 person-years (consistent with incidence rates re- 
Table 1. Baseline Characteristics for Study Sample $(\mathrm{N}=9,451)$

According to Stroke Outcome: Canadian Study of Health and Aging

\begin{tabular}{|c|c|c|c|}
\hline Characteristic & $\begin{array}{l}\text { No Stroke } \\
(\mathrm{n}=\mathbf{8 , 5 7 8})\end{array}$ & $\begin{array}{l}\text { Nonfatal Incident Stroke } \\
\quad(\mathrm{n}=701)\end{array}$ & $\begin{array}{l}\text { Fatal Incident Stroke } \\
\qquad(\mathrm{n}=172)\end{array}$ \\
\hline \multicolumn{4}{|l|}{ Age, $\%$} \\
\hline $65-75$ & 65.7 & 68.5 & $33.3^{*}$ \\
\hline $76-85$ & 27.7 & 27.7 & $43.5^{*}$ \\
\hline$\geq 86$ & 6.6 & 3.8 & $23.2^{*}$ \\
\hline \multicolumn{4}{|l|}{ Sex, \% } \\
\hline Male & 41.5 & 42.7 & 37.6 \\
\hline Female & 58.5 & 57.3 & 62.4 \\
\hline \multicolumn{4}{|l|}{ Race, \% } \\
\hline White & 96.8 & 96.8 & 97.7 \\
\hline Nonwhite & 3.2 & 3.2 & 2.3 \\
\hline \multicolumn{4}{|l|}{ Education, \% } \\
\hline$<$ High school & 63.1 & 65.2 & $71.2^{*}$ \\
\hline High school diploma & 27.1 & 26.2 & 23.8 \\
\hline College degree & 9.8 & 8.6 & $5.0^{*}$ \\
\hline \multicolumn{4}{|l|}{ Marital status, \% } \\
\hline Married & 54.5 & 55.3 & $41.8^{*}$ \\
\hline Not married & 45.5 & 44.7 & $58.2^{*}$ \\
\hline \multicolumn{4}{|l|}{ Health conditions, \% } \\
\hline Heart disease & 26.1 & $31.2^{*}$ & 26.3 \\
\hline Hypertension & 32.0 & $43.4^{*}$ & 39.3 \\
\hline Diabetes mellitus & 9.2 & $15.1^{*}$ & 9.2 \\
\hline Parkinson's disease & 0.9 & 1.4 & $2.7^{*}$ \\
\hline \multicolumn{4}{|l|}{ Cognitive impairment, \% } \\
\hline Yes & 18.0 & 12.8 & $39.5^{*}$ \\
\hline No & 82.0 & 87.2 & $60.5^{*}$ \\
\hline \multicolumn{4}{|l|}{ Physical disability } \\
\hline $\begin{array}{l}\text { Number of activity of daily living disabilities, } \\
\text { mean } \pm \text { standard deviation }\end{array}$ & $0.95 \pm 1.93$ & $0.89 \pm 1.84^{*}$ & $1.69 \pm 1.90^{*}$ \\
\hline
\end{tabular}

${ }^{*}$ Statistically different from nonstroke group $(P<.05)$.

ported elsewhere $\left.{ }^{23,27}\right)$. The majority $(66 \%)$ of the nonfatal incident strokes were documented in the clinical examination, whereas self-report was the most common method for determining that a stroke had not occurred. Health conditions, including hypertension, heart disease, and diabetes mellitus, were more prevalent in those with nonfatal incident stroke than in those with no stroke or fatal incident stroke, whereas Parkinson's disease was more common in those with a fatal incident stroke than in those with no stroke or nonfatal incident stroke. Approximately $4.6 \%$ of all study deaths were due to stroke.

Table 2 reports the results from the multinomial logistic regression analyses for the two stroke outcomes (no stroke is the reference group). Model 1 presents the logistic regression coefficients and corresponding odds ratios (ORs) for the time-invariant sociodemographic characteristics as they relate to nonfatal incident stroke and fatal incident stroke. Older age is a strong risk factor for fatal stroke, with the OR for fatal incident stroke almost seven times as high in those aged 86 and older at baseline as in those aged 65 to 75. As a result of this high mortality risk, adults aged 75 and older were less likely to have a nonfatal incident stroke (Model 1, Table 2). Individuals referred to a clinical exam- ination were significantly more likely than those providing self-reported data to have had a nonfatal incident stroke than no stroke (probably because lower poststroke cognitive dysfunction was the trigger for referral to a clinical examination), but the method of stroke assessment did not modify the effects of age or any of the other important predictor variables in the analyses. Lower education conveys a greater risk for fatal stroke, with $95 \%$ higher odds in those with less than a high school education than in those with a college degree. No significant differences were found according to sex or race, but a strong period effect indicates that the risk of nonfatal and especially fatal incident stroke decreased dramatically over time, net of the effects of age.

Model 2 added time-varying marital status, which had little effect on stroke incidence. Model 3 added the important health risk factors. With the exception of hypertension, health conditions were not significantly associated with the risk of fatal incident stroke (Model 3, Table 2), but all four health conditions were positively associated with greater risk of nonfatal stroke at a subsequent survey wave. Individuals with hypertension and diabetes mellitus had roughly $50 \%$ greater odds of incident stroke at follow-up, whereas heart disease was associated with $39 \%$ higher 


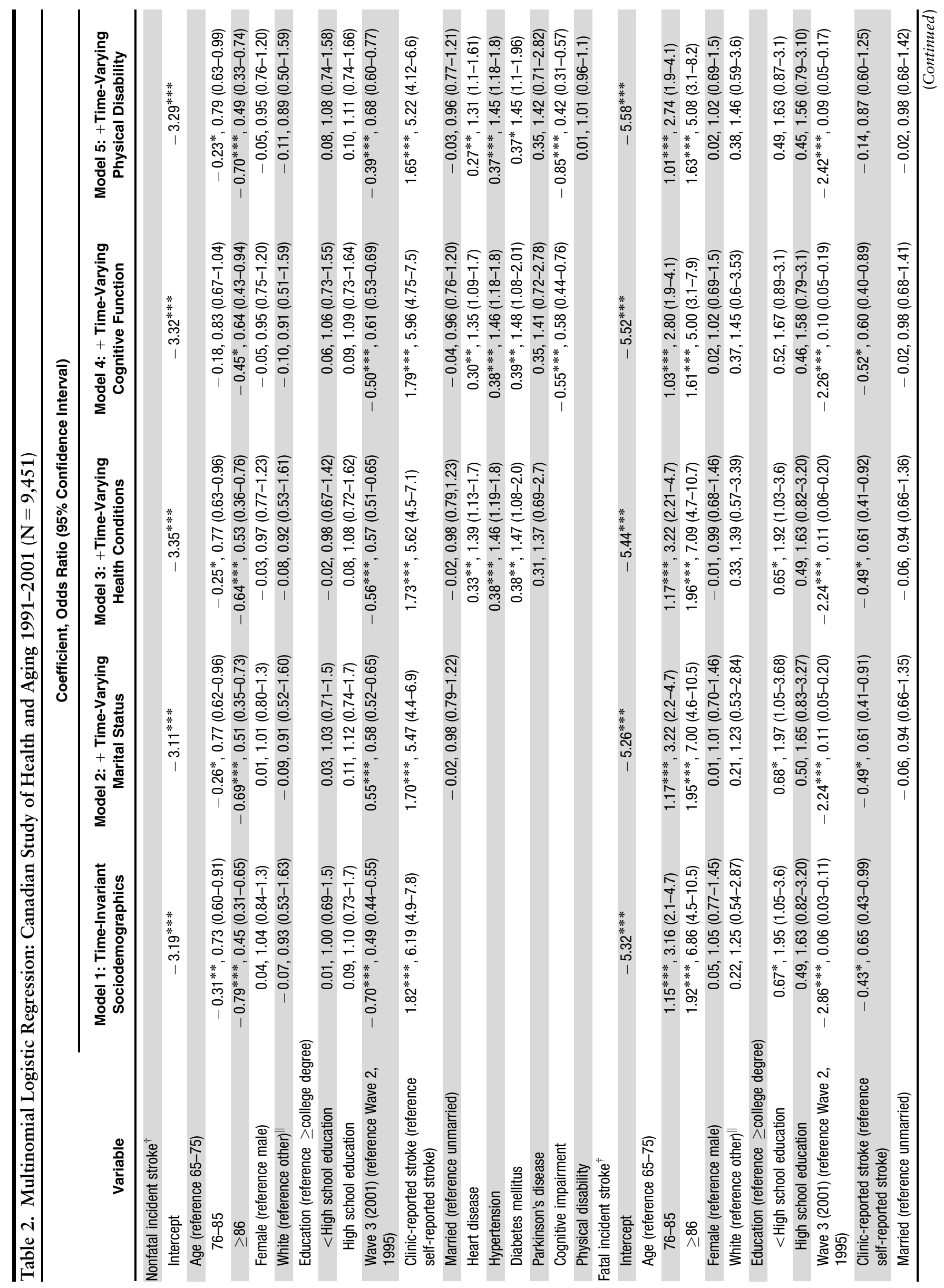


odds. Consistent with the literature, ${ }^{12}$ Parkinson's disease was positively associated with greater risk of stroke, but the rarity of this condition leads to wide confidence intervals around this estimate.

Model 4 added the time-varying indicator of cognitive status; individuals with cognitive impairment had almost twice the odds of fatal incident stroke by a subsequent wave, controlling for other health conditions and sociodemographic risk factors (Table 2). Cognitive impairment accounted for $22 \%$ of the education effect $((0.67-0.52) /$ $0.67=0.25$, Model 1 to Model 4, Table 2) and explained $17 \%$ of the health-adjusted age effect $((1.96-1.61) /$ $1.96=0.17$, Model 3 to Model 4, Table 2) between those aged 86 and older and those aged 65 to 75 . As a result of this strong stroke mortality risk, cognitive impairment was negatively associated with nonfatal stroke. Physical disability had no effect on the odds of stroke at a subsequent wave (Model 5, Table 2).

\section{DISCUSSION}

With a nationally representative cohort of older Canadians, the CSHA provides a unique opportunity to examine the risk factors for incident stroke in later life. Although several longitudinal studies have examined risk factors for stroke, ${ }^{21,23}$ there has not been an attempt to examine the different risk factors for fatal and nonfatal incident stroke in a national population-based sample that includes community-dwelling and institutionalized older adults. This study provided the opportunity to assess the effect of risk factors, including clinically diagnosed cognitive impairment, that are more common in older than younger adults.

Consistent with declining stroke rates in developed countries, ${ }^{28}$ results from this representative cohort indicate that the risks for fatal and nonfatal incident stroke in older adults have been declining since the study began in 1991 . The protective period effect is particularly strong for fatal incident stroke, probably because of the introduction of thrombolytic agents to treat acute stroke over this period. ${ }^{29}$

Stroke risk factors identified in this prospective cohort of older Canadians, including older age, hypertension, heart disease, and diabetes mellitus, are similar to those reported in other studies. ${ }^{6,8}$ In addition, cognitive impairment was a significant independent risk factor for fatal incident stroke in these older adults. Consistent with results from other elderly samples, ${ }^{20,21,23}$ cognitive impairment may be associated with incident stroke because it captures a preclinical phase of "silent" or lacunar strokes (associated with vascular-related cognitive deficits) that predict more-serious subsequent strokes that are more likely to be fatal. ${ }^{3}$ Significant educational disparities in incident fatal stroke exist in this population-based cohort, and consistent with observed associations in the literature between lower education and dementia, ${ }^{30}$ lower cognitive function in the less-educated participants partly explained the educational disparities in stroke in this study.

In contrast to other results, ${ }^{16}$ no relationship was found between physical disability and stroke incidence, perhaps because a measure of difficulty with ADLs was relied on, rather than difficulty with functional performance (e.g., climbing stairs), which was used in other work. ${ }^{16}$ This is a 
potentially important subject that could be investigated further with secondary data from other national surveys.

\section{Limitations}

Although the comprehensive clinical assessments of cognitive function and the nationally representative older sample are major strengths of this analysis, a lack of data on other stroke risk factors, including smoking, alcohol use, obesity, and depression, limited this study. ${ }^{8}$ To some extent, the dummy variable for clinically reported stroke captured the effects of these unmeasured confounders (because lesshealthy people tended to be referred to the clinical examination), but they could potentially attenuate the results. In addition, information on stroke incidence in the community-dwelling sample was self-reported based on experiencing a stroke, or the effects of stroke, in the past year. Although self-reports of stroke have been found to be valid in other population-based surveys, ${ }^{27}$ more-mild strokes (without any residual sequelae) might be missed using this method. Information on stroke subtype (hemorrhage or infarct) was not available in the survey data, and information from death certificates is not always accurate. To ascertain causal direction and minimize recall bias, the analyses were restricted to time-varying risk factors documented at the survey wave before stroke onset. As a result, the effects of risk factors that progress or emerge between waves might have been underestimated.

In spite of these limitations, this study highlights the importance of cognitive impairment as an important risk factor for fatal stroke in older adults. This finding provides further evidence of the need for targeted screening for cognitive impairment in older adults, even those without preexisting clinical conditions. Early intervention in older adults with cognitive deficits may decrease the incidence of fatal stroke in later life.

\section{ACKNOWLEDGMENTS}

Conflict of Interest: The editor in chief has reviewed the conflict of interest checklist provided by the authors and has determined that the authors have no financial or any other kind of personal conflicts with this paper.

Financial Disclosure: The data reported in this article were collected as part of the CSHA. The core study was funded by the Seniors' Independence Research Program, through the National Health Research and Development Program (NHRDP) of Health Canada (Project 66063954-MC(S)). Additional funding was provided by Pfizer Canada, Inc. through the Medical Research Council/Pharmaceutical Manufacturers Association of Canada Health Activity Program, NHRDP (Project 6603-1417-302(R)), Bayer Inc., and the British Columbia Health Research Foundation (Project 38(93-2) and 34(96-1)). The study was coordinated through the University of Ottawa and the Division of Aging and Seniors, Health Canada. We gratefully acknowledge the support of the Toronto Rehabilitation Institute Foundation, the Ontario Work study program, and a grant to the Toronto Rehabilitation Institute from the Ontario Ministry of Health and Long Term Care.

Author Contribution: Philippa Clarke and Vanessa Blount prepared the manuscript and analyzed and interpreted the data. Angela Colantonio assisted with the prep- aration of the manuscript and with the analysis and interpretation of the data.

Sponsor's Role: The sponsors had no role in the analysis and preparation of the manuscript.

\section{REFERENCES}

1. Rosamond W, Flegal K, Friday G et al. Heart disease and stroke statistics-2007 update: A report from the American Heart Association Statistics Committee and Stroke Statistics Subcommittee. Circulation 2007;115:e69-e171.

2. Neyer JR, Greenlund KJ, Denny CH et al. Prevalence of stroke - United States, 2005. MMWR Morb Mortal Wkly Rep 2007;56:469-474.

3. Chen R-L, Balami JS, Esiri MM et al. Ischemic stroke in the elderly: An overview of evidence. Nat Rev Neurol 2010;6:256-265.

4. Brocklehurst JC, Morris P, Andrews K. Social effects of stroke. Soc Sci Med 1981;15:35-39.

5. Clarke P, Marshall VW, Black SE et al. Well-being following stroke in Canadian seniors: Findings from the Canadian Study of Health and Aging. Stroke 2002;33:1016-1021.

6. Lindenstrøm E. Influence of systolic and diastolic blood pressure on stroke risk: A prospective observational study. Am J Epidemiol 1995;142:1279-1290.

7. Wolf PA, D'Agostino RB, Kannel WB et al. Cigarette smoking as a risk factor for stroke. JAMA 1988;259:1025-1029.

8. O'Donnell MJ, Xavier D, Liu L et al. Risk factors for ischaemic and intracerebral haemorrhagic stroke in 22 countries (the INTERSTROKE study): A case-control study. Lancet 2010;376:112-123.

9. Abbott RD. Physical activity in older middle-aged men and reduced risk of stroke: The Honolulu Heart Program. Am J Epidemiol 1994;139:881-893.

10. Kannel WB, Wolf PA, Verter J. Manifestations of coronary disease predisposing to stroke. JAMA 1983;250:2942-2946.

11. Gill JS, Zezulka AV, Shipley MJ et al. Stroke and alcohol consumption. N Engl J Med 1986;315:1041-1046.

12. Becker C, Jick SS, Meier CR. Risk of stroke in patients with idiopathic Parkinson disease. Parkinsonism Relat Disord 2010;16:31-35.

13. National Institutes of Health. Incidence and Prevalence: 2006 Chart Book on Cardiovascular and Lung Diseases. Bethesda, MD: National Heart, Lung, and Blood Institute, 2006.

14. Avendano M, Kawachi I, Van Lenthe F et al. socioeconomic status and stroke incidence in the US elderly: The role of risk factors in the EPESE study. Stroke 2006;37:1368-1373.

15. Andrepetersson L, Engstrom G, Hedblad B et al. Social support at work and the risk of myocardial infarction and stroke in women and men. Soc Sci Med 2007;64:830-841.

16. Colantonio A. Level of function predicts first stroke in the elderly. Stroke 1992;23:1355-1357.

17. Arboix A, Garca-Eroles L, Massons J. Acute stroke in very old people: Clinical features and predictors of in-hospital mortality. J Am Geriatr Soc 2000;48:3641.

18. Woo J, Lau E, Kay R. Elderly subjects aged 70 years and above have different risk factors for ischemic and hemorrhagic strokes compared to younger subjects. J Am Geriatr Soc 1992;40:124-129.

19. Rojas JI, Zurrú MC, Romano M et al. Acute ischemic stroke and transient ischemic attack in the very old - risk factor profile and stroke subtype between patients older than 80 years and patients aged less than 80 years. Eur J Neurol 2007;14:895-899.

20. Ostir GV, Raji MA, Ottenbacher KJ et al. Cognitive function and incidence of stroke in older Mexican Americans. J Gerontol A Biol Sci Med Sci 2003;58A:M531-M535.

21. Ferrucci L, Guralnik JM, Salive ME et al. Cognitive impairment and risk of stroke in the older population. J Am Geriatr Soc 1996;44:237-241.

22. Alves de Moraes S, Szklo M, Tilling K et al. Cognitive functioning as a predictor of ischemic stroke incidence. Epidemiology 2003;14:673-679.

23. DeFries T, Avendaño M, Glymour MM. Level and change in cognitive test scores predict risk of first stroke. J Am Geriatr Soc 2009;57:499-505.

24. Elkins JS. Cognitive function predicts first-time stroke and heart disease. Neurology 2005;64:1750-1755.

25. Teng EL, Chui HC. The Modified Mini-Mental State (3MS) Examination. J Clin Psychiatry 1987;48:314-318.

26. Tierney MC, Yao C, Kiss A et al. Neuropsychological tests accurately predict incident Alzheimer disease after 5 and 10 years. Neurology 2005;64:18531859.

27. Glymour MM, Avendano M. Can self-reported strokes be used to study stroke incidence and risk factors?: Evidence from the Health and Retirement Study. Stroke 2009;40:873-879. 
28. Feigin VL, Lawes CMM, Bennett DA et al. Worldwide stroke incidence and early case fatality reported in 56 population-based studies: A systematic review. Lancet Neurol 2009;8:355-369.

29. Grotta J, Marler J. Intravenous rt-PA: A tenth anniversary reflection. Surg Neurol 2007;68:S12-S16.
30. Stern Y, Gurland B, Tatemichi TK et al. Influence of education and occupation on the incidence of Alzheimer's disease. JAMA 1994;271:1004-1010. 